\title{
3D printable gelatin hydrogels incorporating graphene oxide to enable spontaneous myogenic differentiation
}

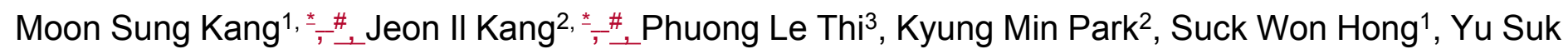

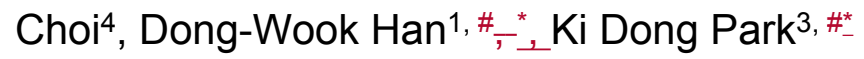

${ }^{1}$ Department of Cogno-Mechatronics Engineering, Pusan National University, Busan 46241, Republic of Korea

${ }^{2}$ Department of Bioengineering and Nano-bioengineering, Incheon National University, Incheon 22012, Republic of Korea

${ }^{3}$ Department of Molecular Science and Technology, Ajou University, Suwon 16499, Republic of Korea ${ }^{4}$ School of Human Sciences, The University of Western Australia, WA 6009, Australia

\#-Co-correspondence to:

Dong-Wook Han, Department of Cogno-Mechatronics Engineering, Pusan National University, Busan 46241, Republic of Korea. Tel: +82-51-510-7725; E-mail address: nanohan@pusan.ac.kr

And

Ki Dong Park, Department of Molecular Science and Technology, Ajou University, Suwon 16499, Republic of Korea. Tel.: +82-31-219-1846; E-mail address: kdp@ajou.ac.kr 


\section{Materials and methods}

\subsection{Fabrication of GO-incorporating hydrogel (GO/GHPA) bioinks}

GHPA hydrogels were synthesized as reported previously ${ }^{1,2}$. Briefly, 3-(4-hydroxyphenyl) propionic acid (HPA, Sigma-Aldrich, MO, USA) was dissolved in distilled water (DW)/dimethylformamide (Junsei, Japan) 3:2 co-solvents and reacted in 1-ethyl-3-(3-dimethylaminopropyl)-carbodiimide/Nhydroxysuccinimide (Sigma-Aldrich, MO, USA) for 15 minutes to activate the carboxyl groups. The activated solution was reacted with gelatin (type A from porcine skin, less than 300 bloom, SigmaAldrich, MO, USA) dissolved in DW at $40{ }^{\circ} \mathrm{C}$ for $24 \mathrm{~h}$. For purification, the resulting solution was treated with a $\mathrm{NaCl}$ solution and DW/ethanol, and then filtered and lyophilized to produce the GHPA hydrogel. The degree of substitution of the HPA was measured using a UV spectrometer (Jasco, V-750 UV/vis/NIR, Tokyo, Japan). The GHPA polymer was dissolved in DW $(1 \mathrm{mg} / \mathrm{mL})$, and the absorbance was measured at a $275 \mathrm{~nm}$ wavelength.

GO/GHPA bioink was prepared as follows. The GHPA hydrogel was sterilized with UV for 10 minutes, which was dissolved in sterilized phosphate-buffered saline (PBS; Gibco, Paisley, United Kingdom) at 3 wt $\%$ at $37^{\circ} \mathrm{C}$ for 4 h. A 100 ppm GO solution (Sigma-Aldrich, MO, USA) was added to the dissolved GHPA hydrogel at a volume ratio of 1:9, and the GO/GHPA pre-gel was divided into two aliquots. HRP and GOx were added to the A solution (GO/GHPA:HRP:GOx = 8:1:1), and $\mathrm{H}_{2} \mathrm{O}_{2}$ and glucose (Glc) were added to the $B$ solution (GO/GHPA: $\left.\mathrm{H}_{2} \mathrm{O}_{2}: \mathrm{GlC}=8: 1: 1\right)$. Each of the $\mathrm{A}$ and $\mathrm{B}$ solutions were mixed homogeneously by vortexing and pipetting. Table 1 represents the detailed concentration of each component. 
Table 1. The composition (final concentration) of GO/GHPA hydrogel

\begin{tabular}{ccccccc}
\hline $\begin{array}{c}\text { Sample } \\
\text { code }\end{array}$ & $\begin{array}{c}\mathrm{GHPA} \\
(\mathrm{wt} \%)\end{array}$ & $\begin{array}{l}\mathrm{GO} \\
(\mathrm{ppm})\end{array}$ & $\begin{array}{c}\mathrm{H}_{2} \mathrm{O}_{2} \\
(\mathrm{wt} \%)\end{array}$ & $\begin{array}{c}\mathrm{HRP} \\
(\mathrm{U} / \mathrm{mL})\end{array}$ & $\begin{array}{c}\mathrm{GOx} \\
(\mu \mathrm{U} / \mathrm{mL})\end{array}$ & $\begin{array}{c}\mathrm{Glc} \\
(\mathrm{mM})\end{array}$ \\
\hline GHPA & 2.4 & 0 & 0.0015 & 0.63 & 2.5 & 25 \\
GO/GHPA & 2.4 & 8 & 0.0015 & 0.63 & 2.5 & 25 \\
\hline
\end{tabular}

\subsection{Physicochemical characterization of GO/GHPA bioink}

The internal microscopic images of the GHPA and GO/GHPA hydrogels were obtained by scanning electron microscopy (SEM, JSM-7800F, JEOL Ltd., Tokyo, Japan). The GHPA and GO/GHPA hydrogels were prepared, as mentioned above. They were lyophilized to prevent morphological deformation during the drying process. The lyophilized hydrogels were cross-sectioned and sputtercoated with gold/platinum by ion sputtering. Subsequently, their internal structures were visualized by SEM.

Fourier transform infrared (FT-IR, Nicolet560, Nicolet Co., Madison, USA) spectroscopy and Raman spectroscopy were conducted to evaluate the compositional characteristics of the GHPA and GO/GHPA hydrogels. The FT-IR spectra were recorded in adsorption mode in the wavelength range of $400-4000 \mathrm{~cm}^{-1}$ with a resolution of $1.0 \mathrm{~cm}^{-1}$. The Raman spectra of GHPA and GO/GHPA hydrogels were obtained by Raman spectroscopy (Micro Raman PL Mapping System, Dongwoo Optron Co., Gwangju, Korea) with an Ar-ion laser of wavelength $514.5 \mathrm{~nm}$ at a power of $5 \mathrm{~mW}$.

\subsection{Rheological analysis of GO/GHPA hydrogel}

The viscoelastic properties of GO/GHPA hydrogels were measured using a rheometric fluid spectrometer (DHR-1, TA Instruments, New Castle, DE) in oscillatory mode, as described previously 
3-6. To measure the elastic modulus $\left(\mathrm{G}^{\prime}\right)$ and viscous modulus $\left(\mathrm{G}^{\prime \prime}\right)$ of the hydrogels, $200 \mu \mathrm{L}$ of the

hydrogels were prepared on a $20 \mathrm{~mm}$ plate geometry with a solvent trap wetted with DW. The viscoelastic modulus depending on the GO incorporation was monitored in dynamic time sweep mode at a frequency of $0.1 \mathrm{~Hz}$ and $0.1 \%$ strain at $37^{\circ} \mathrm{C}$.

\subsection{In vitro degradation behaviors of GO/GHPA hydrogel}

The proteolytic degradation of the GO/GHPA hydrogels was determined gravimetrically. The hydrogels $(100 \mu \mathrm{L})$ were prepared in microtubes, and the initial hydrogel weight $\left(W_{i}\right)$ was measured. Subsequently, the hydrogels were immersed in $200 \mu \mathrm{L}$ DPBS with or without $0.01 \mathrm{mg} / \mathrm{mL}$ collagenase at $37^{\circ} \mathrm{C}$, as reported previously $3,4,6$. At predetermined time points, the degraded hydrogels $\left(W_{d}\right)$ were weighed after removing the media from the microtubes. Fresh media were added to the microtubes after weighing. The weight of the remaining hydrogels was calculated using the following equation:

$$
\text { Hydrogel weight }(\%)=\left(W_{d} / W_{i}\right) \times 100
$$

\subsection{Degradation and thermal stability of the bioink}

The degradation of the GHPA and GO/GHPA hydrogel was tested as follows. Culture dishes were pre-weighed with an electronic balance and marked. Subsequently, GO/GHPA hydrogels were loaded into dishes with a $2 \mathrm{~mL}$ solution for each dish and incubated at $37^{\circ} \mathrm{C}$ for $2 \mathrm{~h}$. After gelation, the hydrogelcontaining plates were weighed; $2 \mathrm{~mL}$ PBS was added and incubated for 21 days. At different time points, the hydrogel-containing dishes were weighed after PBS had been removed entirely. The degree of degradation was evaluated by the weight ratio of the initial hydrogel-containing dishes and remaining hydrogels. The thermal stability of the GHPA and GO/GHPA hydrogel was evaluated by thermogravimetric analysis (TGA, DSC 8000, Perkin Elmer, Waltham, MA). The hydrogels were preweighed at the initial state and heated from $\mathrm{RT}$ to $700{ }^{\circ} \mathrm{C}$ at a heating rate of $10{ }^{\circ} \mathrm{C} /$ min under an $\mathrm{N}_{2}$ 
atmosphere.

\subsection{C2C12 myoblasts culture}

The C2C12 myoblasts were purchased from the American Type Culture Collection (ATCC, Rockville, MD) and cultured routinely in a 75-T flask in complete Dulbecco's modified Eagle's medium (DMEM, Welgene, Daegu, Korea) supplemented with 10\% fetal bovine serum (FBS, Welgene, Daegu, Korea) and $1 \%$ penicillin-streptomycin solution (P/S, Sigma-Aldrich, USA). The cells were maintained at $37^{\circ} \mathrm{C}$ in a humidified atmosphere containing $5 \% \mathrm{CO}_{2}$. A subculture was conducted using a trypsin-EDTA solution (Welgene, Daegu, Korea) at $80 \%$ confluence, and the culture media was replaced every $48 \mathrm{~h}$.

\section{7. $3 D$ bioprinting of GO/GHPA}

An extrusion type 3D bioprinter (In-vivo, Rokit, Seoul, Korea) was set up. A lattice cuboid 3D model $(2 \mathrm{~cm} \times 2 \mathrm{~cm} \times 0.2 \mathrm{~cm})$ was produced and sliced into the g-code file using NewcreatorK software (Rokit). The printing parameters, such as printing speed, flow rate, the distance between the nozzle and bed, and $Z$ hop, were optimized before 3D printing. For the minimized shear stress and optimized printability, the nozzle gauge was set to $600 \mu \mathrm{m}$, which showed the most suitable cell viability and printability (data not shown). The temperature of the dispenser and bed was set to $5^{\circ} \mathrm{C}$ to retain the lamination of the pre-gel state bioink. The composite solutions were mixed in a $10 \mathrm{~mL}$ syringe to maintain a pre-gel state during the printing process. Cell pellets were resuspended with prepared pre-gel bioink in $5 \times 10^{5}$ cells $/ \mathrm{mL}$ (live/dead assay), or $10^{5}$ cells $/ \mathrm{mL}$ (myogenic differentiation). In the case of a long period culture (for myogenic differentiation, 8 days), cell density was reduced to $10^{5} \mathrm{cell} / \mathrm{mL}$ to maintain the structural integrity of constructs, considering $\mathrm{H}_{2} \mathrm{O}_{2}$ consumption and enzyme activity change by cellular activities. Four layers were printed by piston-driven pressure $(1 \mathrm{~mm} / \mathrm{min})$ onto cover glasses (Marienfeld, Thuringia, Germany) within $5 \mathrm{~min}$, and printed constructs were transferred to six-well plates. After 30 min. of stabilization in RT, a fresh medium was added with the plate. Before cell-laden printing, 
A Vaseline prototype model and non-cell laden model were printed for a printability evaluation (Fig. S1A-D).

When the bioink is in proper gelation condition, the extruded filament would result in regular grids and square holes in the fabricated lattice structure. Following are common 3D printability quantification methods introduced by Ouyang et al. ${ }^{7}$ If the hydrogel is in under-gelation condition, the printed filament would create circular holes. The circularity (C) of the enclosed area was defined as the following:

$$
C=\frac{4 \pi A}{L^{2}}
$$

For a square-shaped hole, circularity is equal to $\pi / 4$. $L$ and $A$ means perimeter and area of lattice holes, respectively. Printability $(\mathrm{Pr})$ of GO/GHPA hydrogel was assessed as below:

$$
\operatorname{Pr}=\frac{\pi}{4} \cdot \frac{1}{C}=\frac{L^{2}}{16 A}
$$

For an ideal printed construct, the Pr value was 1 and the acceptable printable region was set to 0.9 to 1.1. The larger $\operatorname{Pr}$ value means under-gelation of hydrogel, while the smaller $\operatorname{Pr}$ value means over-gelation of the hydrogel. Optical images of Fig. S1E were analyzed by ImageJ software to determine the perimeter and area of lattice holes (Fig. S1F).

\subsection{Cell viability and proliferation}

Cell viability and proliferation were determined using a Live/dead viability kit (Molecular Probes, Eugene, OR). After 1, 3, 5, and 7 days of culture, the cell-loaded hydrogels were incubated with a live and dead cell assay solution ( $2 \mu \mathrm{M}$ calcein $\mathrm{AM}$ and $4 \mu \mathrm{M}$ ethidium homodimer- 1 ) for 30 min in the dark at room temperature. The stained samples were observed using a fluorescence microscope (IX 81 , Olympus, Tokyo, Japan) and a confocal microscope (LSM 800, Zeiss, Oberkochen, Germany). The fluorescence signals from the live and dead cells were quantified using Image J software $(\mathrm{NIH}$, Bethesda, 
MD) to evaluate cell viability and proliferation.

\subsection{Myogenic differentiation and immunostaining}

GO/GHPA hydrogel constructs were cultured in growth media for 8 days. For comparison as a positive control, the GHPA hydrogel medium was changed to a differentiation medium (DMEM supplemented with $2 \% \mathrm{HS}$, and $1 \% \mathrm{P} / \mathrm{S}$; HS: horse serum, P/S: pen strep, Welgene, Daegu, Korea) after $24 \mathrm{~h}$ culture in the growth medium. Fresh medium was added every $48 \mathrm{~h}$. The differentiation of encapsulated cells was evaluated by immunofluorescence staining. After 8 days of culture, the cells were fixed with 3.7\% paraformaldehyde (Sigma-Aldrich, MO, USA) for $10 \mathrm{~min}$. After fixation, the cells were permeabilized and blocked with a $0.1 \%$ Triton X-100 (Sigma-Aldrich Co.) solution for 5 min, and $2 \%$ bovine serum albumin (Sigma-Aldrich, MO, USA) solution for 30 min. Anti-myosin heavy chain (MHC) mouse monoclonal antibodies $(500 \mathrm{ng} / \mathrm{mL})$ were added and reacted at $4{ }^{\circ} \mathrm{C}$ in a refrigerator overnight. After reacting overnight, the cells were incubated with FITC-labelled goat anti-mouse IgG antibody at 1:1000 PBS (Abcam, Cambridge, MA) and $165 \mathrm{nM}$ TRITC-labeled phalloidin (Molecular probes, Eugene, OR) for $30 \mathrm{~min}$ in the dark at room temperature. The nuclei were counterstained with 300 nM 4',6-diamidino-2-phenylindole (Sigma-Aldrich, MO, USA) under the same conditions. During every process, the samples were washed twice with PBS. For fluorescence microscopy, fluorescence microscope and confocal microscope were utilized. The fluorescence signals from the confocal microscopy were quantified using ImageJ software to evaluate f-actin alignment and ratio of $\mathrm{MHC}$ positive area.

\subsection{Western blotting}

After incubation in hydrogels for 8 days, the constructs were dissolved in $55 \mathrm{mM}$ sodium citrate and $20 \mathrm{mM}$ EDTA in $0.9 \% \mathrm{NaCl}$ and stirred using a pipette for $10 \mathrm{~min}$ at RT to allow cell isolation. The C2C12 myoblasts were washed twice with cold PBS (10 mM, pH 7.4) and ice-cold RIPA lysis buffer 
(Santa Cruz Biotechnology Inc., Santa Cruz, CA) was then added. After 5 min, the cells were scraped, and the lysate was centrifuged at $14,000 \times \mathrm{g}$ for $20 \mathrm{~min}$ at $4{ }^{\circ} \mathrm{C}$. The proteins were extracted from the total lysate, and the protein concentration was determined using a $\mathrm{BCA}^{\mathrm{TM}}$ protein assay according to the manufacturer's protocol (Pierce, Rockford, IL). For immunoblot analysis, equal amounts of protein (40 $\mu \mathrm{g})$ were run on a 4/20 polyacrylamide-SDS gel (Daiichi Pure Chemicals Co, Ltd, Tokyo, Japan) for $1 \mathrm{~h}$ at $30 \mathrm{~mA}$ and blotted onto a PVDF membrane for $50 \mathrm{~min}$ at $35 \mathrm{~mA}$. The blots were blocked with a blocking buffer (Nacalai Tesque Inc., Kyoto, Japan) for $1 \mathrm{~h}$ at $25^{\circ} \mathrm{C}$ and incubated with rabbit polyclonal anti-MyoD and mouse monoclonal anti-myogenin Abs at 1:500 dilutions. As a reference, a mouse monoclonal Ab to glyceraldehyde-3-phosphate dehydrogenase (GAPDH) (Abcam, Cambridge, MA) was used at 1: 5,000 dilutions overnight at $4{ }^{\circ} \mathrm{C}$. The blots were incubated with either goat antirabbit (Santa Cruz Biotechnology Inc., Santa Cruz, CA) or goat anti-mouse (Amersham Biosciences, Buckinghamshire, England) secondary Ab, horseradish peroxidase-conjugated at 1: 2,000 dilutions. Protein expression was detected using a Chemilumi-one chemiluminescent kit (Nacalai Tesque Inc.) and X-ray film (Fujifilm, Tokyo, Japan).

\subsection{Statistical analysis}

All variables were tested in three independent cultures for each experiment, which was repeated once or twice $(n=3-6)$. The quantitative data are expressed as the mean \pm standard deviation (SD). The data were tested for the homogeneity of the variances using the Levene test, before statistical analysis. Statistical comparisons were carried out using a one-way analysis of variance (ANOVA; SAS Institute Inc., Cary, NC), followed by a Bonferroni test for multiple comparisons. A $p$ value $<0.05$ was considered statistically significant. 


\section{Supplementary figures}

(A)

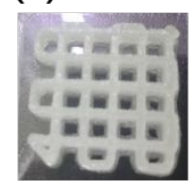

(C)
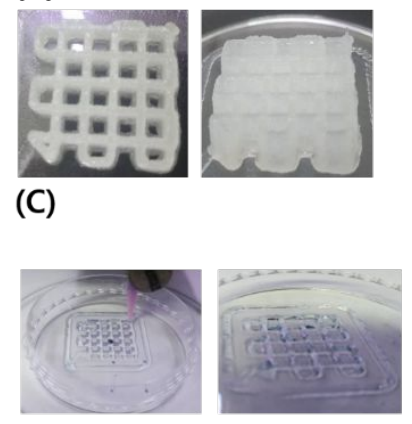

(B)

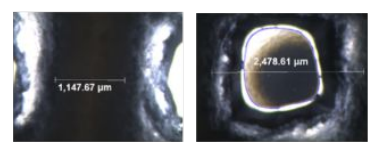

(D)

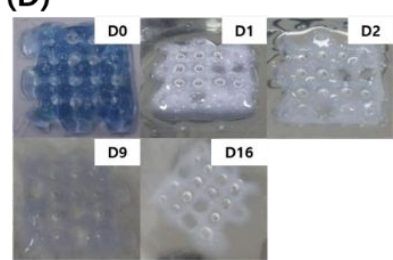

(E)

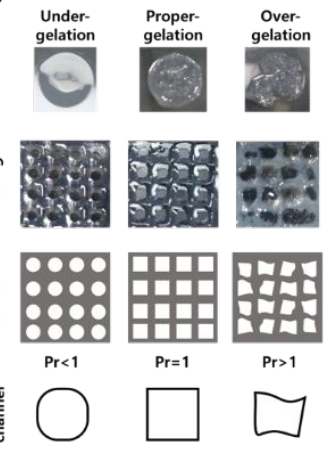

(F)

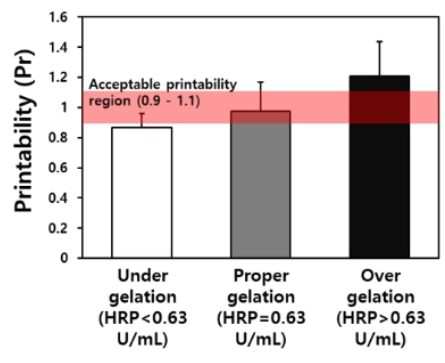

Figure. S1. Printability evaluation. (A) Digital image of prototype model printed with Vaseline. (B) Rough scaling of Vaseline prototype by optical microscopy. Scale bars represent $1,147.67 \mu \mathrm{m}$ and 2,478.61 $\mu \mathrm{m}$, respectively. (C) Digital images of the 3D printing process of GO/GHPA hydrogel. $10 \mu \mathrm{L} / 1.5 \mathrm{~mL}$ of Trypan blue was incorporated into the hydrogel to be easily recognized. (D) Structural stability of printed constructs in DPBS at culture condition. D0 D16 represent DIV (day in vitro) $0 \sim 16$. Hydrogel degradation at each time point was observed by the naked eye and recorded by digital images. (E) Printability was assessed by the degree of gelation. (F) Printability of GO/GHPA hydrogel by different HRP concentration. The results in $(F)$ are shown as the mean $\pm S D(n=16)$.

(A)

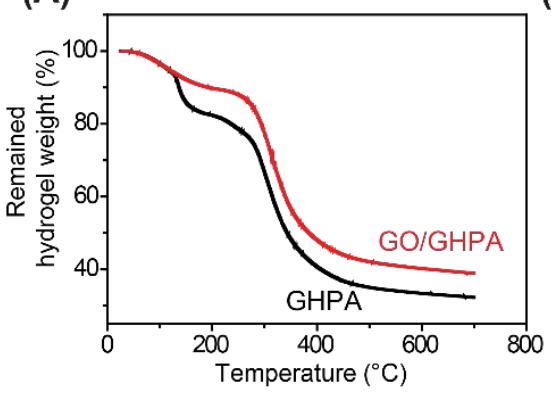

(D)

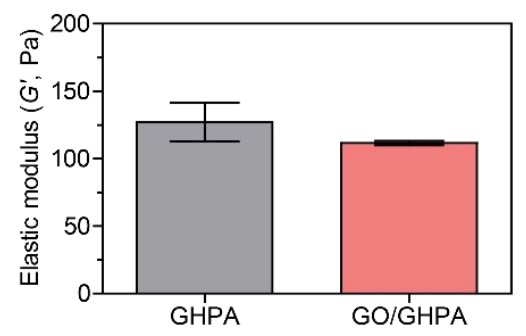

(B)

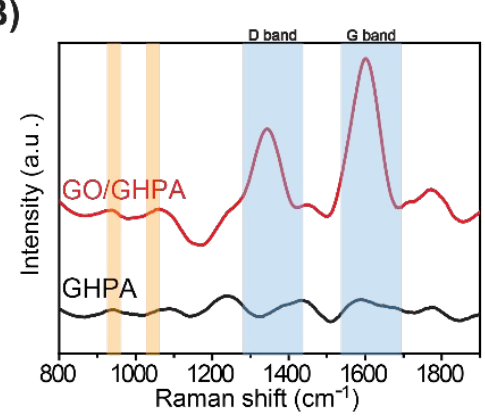

(E)

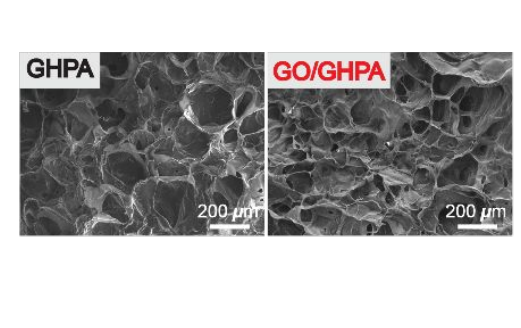

(C)

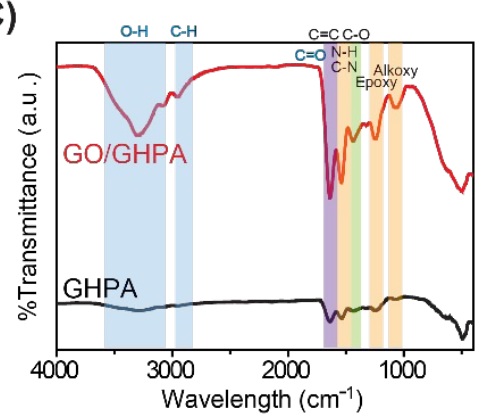

(F)

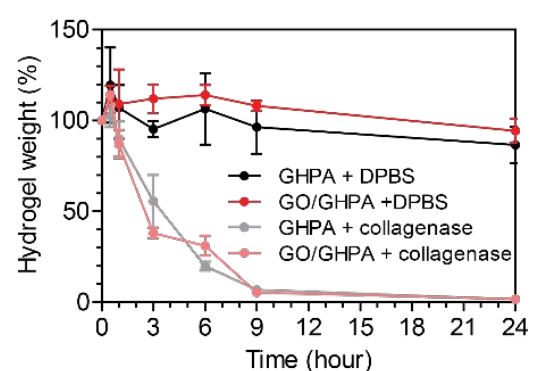

Figure. S2. Physicochemical analysis of GO/GHPA hydrogel matrices. (A) TGA profile, (B) Raman spectra, (C) FT-IR spectra, (D) Elastic properties, (E) Morphologies of dehydrated hydrogel matrices, and (F) in vitro proteolytic degradation. The scale bars represent $200 \mu \mathrm{m}$. The results in (D and F) are shown as the mean \pm SD $(n=3)$.
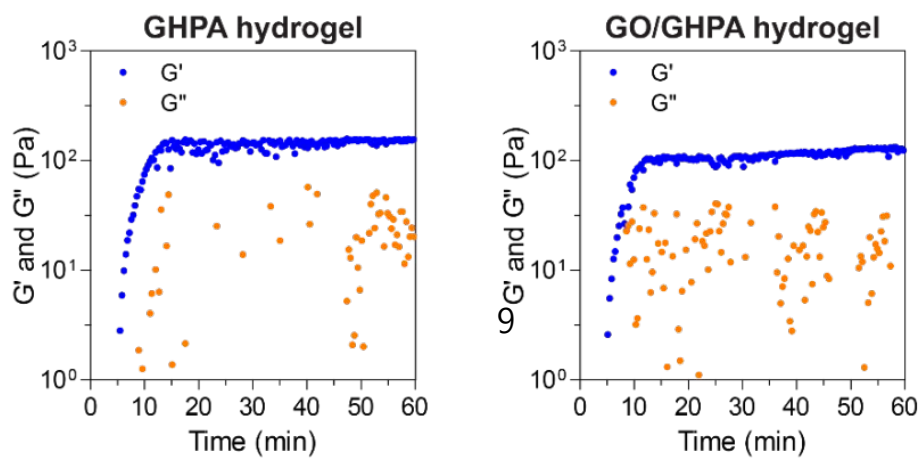
Figure. S3. The viscoelastic properties of GO/GHPA hydrogels. The elastic modulus $\left(G^{\prime}\right)$ and viscous modulus $\left(G^{\prime \prime}\right)$ of the GO/GHPA hydrogels were measured in oscillatory mode using a rheometric fluid spectrometer.

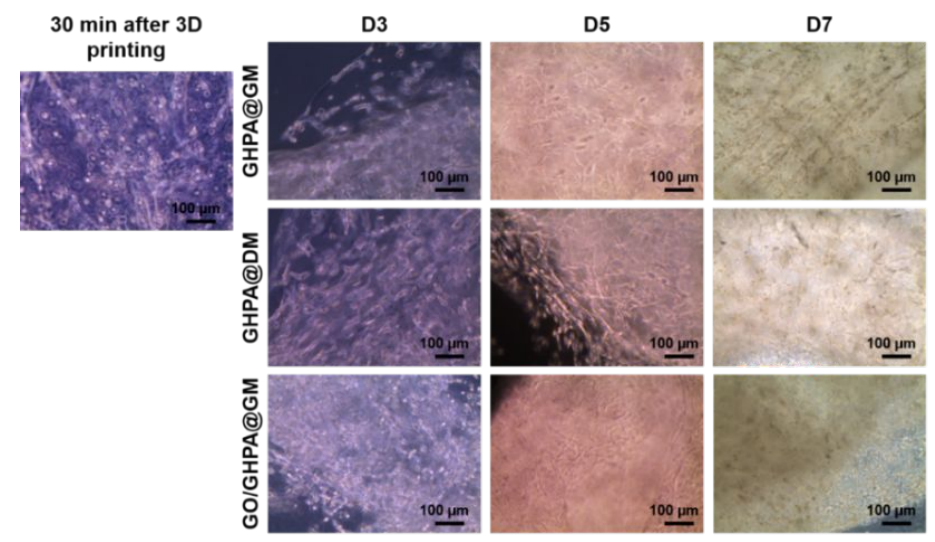

Figure. S4. Morphology of $\mathrm{C} 2 \mathrm{C} 12$ cells in hydrogels. Morphology of laden $\mathrm{C} 2 \mathrm{C} 12$ cells was observed by optical microscopy. Cells showed spherical morphology after printing, but changed into fibroblastic and mesh-like morphology during cultured. D3, D5 and D7 represent DIV (day in vitro) 3, 5 and 7, respectively. Scale bars represent $100 \mu \mathrm{m}$.

\section{[The video is attached separately]}

Movie S1. 3D image of C2C12 cell-laden GO/GHPA hydrogels cultured in GM. The 3D image was obtained by z-stack imaging of confocal microscopy. Green, red and blue fluorescence represent MHC, f-actin, and nucleus, respectively.

\section{References}

(1). Kim, B. Y.; Lee, Y.; Son, J. Y.; Park, K. M.; Park, K. D., Dual Enzyme-Triggered In Situ Crosslinkable Gelatin Hydrogels for Artificial Cellular Microenvironments. Macromol. Biosci. 2016, 16 (11), 1570-1576.

(2). Lee, Y.; Choi, K. H.; Park, K. M.; Lee, J. M.; Park, B. J.; Park, K. D., In situ forming and $\mathrm{H}_{2} \mathrm{O}_{2}$-releasing hydrogels for treatment of drug-resistant bacterial infections. ACS Appl. Mater. Interfaces 2017, 9 (20), 16890-16899.

(3). Park, K. M.; Gerecht, S., Hypoxia-inducible hydrogels. Nat. Commun. 2014, 5 (1), 1-12.

(4). Park, S.; Park, K. M., Hyperbaric oxygen-generating hydrogels. Biomaterials 2018, 182, 234-244.

(5). Kang, J. I.; Park, K. M.; Park, K. D., Oxygen-generating alginate hydrogels as a bioactive acellular matrix for facilitating wound healing. J. Ind. Eng. Chem. 2019, 69, 397-404.

(6). Le Thi, P.; Lee, Y.; Tran, D. L.; Thi, T. T. H.; Kang, J. I.; Park, K. M.; Park, K. D., In situ forming and reactive oxygen species-scavenging gelatin hydrogels for enhancing wound healing efficacy. Acta Biomater. 2020, 103, 142152.

(7). Ouyang, L.; Yao, R.; Zhao, Y.; Sun, W. J. B., Effect of bioink properties on printability and cell viability for 3D bioplotting of embryonic stem cells. Biofabrication 2016, 8 (3), 035020. 